\section{Anaesthetic Management of Subglottic Foreign Body}

\section{Shalini Subramanian* and Ravindra Madhavi}

Department of Paediatric Anaesthesia, Indira Gandhi Institute of Child Health, Bengaluru, India

\begin{abstract}
Subglottic foreign bodies pose an anaesthetic challenge in view of their impending or existing airway obstruction requiring urgent rigid bronchoscopy and removal. Here, we present the case of a nine year old boy who had aspirated a whistle. The clinical signs, diagnosis and management of a subglottic foreign body are discussed.

Keywords: Foreign body; Subglottic
\end{abstract}

Key messages: Subglottic foreign bodies need rigid bronchoscopy and removal. The anaesthetic management is discussed.

\section{Introduction}

Subglottic foreign bodies pose an anaesthetic challenge. They constitute about $8 \%$ of all tracheobronchial foreign bodies [1]. Subglottic foreign bodies need urgent management as their mere presence in that area indicates their large size or adherence to the laryngotracheal wall. They may be removed by direct laryngoscopy, rigid bronchoscopy or tracheotomy.

\section{Case}

A nine year old boy weighing $18 \mathrm{~kg}$ presented for rigid bronchoscopy and Foreign Body (FB) removal. He had aspirated a whistle 8 days earlier following which he developed a cough that had subsided after a day. His only complaint was hoarseness of voice since the FB aspiration. He was being treated for FB of the digestive tract and was referred to a tertiary care centre when he had a violent bout of cough and expelled a portion of the FB. On examination he had no respiratory distress or stridor. There was a mild whistling sound during expiration on auscultation over the trachea. His respiratory rate was 16/minute and room air $\mathrm{SpO}_{2}$ was $99 \%$. Careful examination of the radiograph of neck and chest showed no abnormality.

The child arrived in the operating room with an intravenous line in situ where standard monitors including ECG, $\mathrm{SpO}_{2}$ and NIBP were

*Corresponding author: Shalini Subramanian, Department of Paediatric Anaesthesia, Indira Gandhi Institute of Child Health, South Hospital Complex, Bengaluru, India, Tel: +91 7406610222; E-mail: drshalinisubramanian@gmail.com

Citation: Subramanian S, Madhavi R (2015) Anaesthetic Management of Subglottic Foreign Body. J Clin Stud Med Case Rep 2: 024.

Received: August 13, 2015; Accepted: October 06, 2015; Published: October 23, 2015 applied. He was premedicated with intravenous dexamethasone 2 $\mathrm{mg}$, hydrocortisone $40 \mathrm{mg}$, glycopyrolate $0.1 \mathrm{mg}$ and fentanyl $40 \mu \mathrm{g}$. Anaesthesia was induced intravenously with propofol $80 \mathrm{mg}$ in aliquots of $20 \mathrm{mg}$ and an infusion of propofol was started at $8 \mathrm{mg} /$ $\mathrm{kg} /$ hour. He was allowed to breathe $100 \% \mathrm{O}_{2}$ through a face mask using a Jackson Rees circuit. His heart rate was 110 per minute, blood pressure $84 / 56 \mathrm{mmHg}$ and saturation $98 \%$. Direct laryngoscopy was done and the vocal cords sprayed with $10 \mathrm{mg}$ of $2 \%$ xylocaine. Injection lidocaine $20 \mathrm{mg}$ was given intravenously to reduce the response to intubation and after 60 seconds, the child was positioned with a shoulder roll and direct laryngoscopy was done by the pediatric surgeon who identified a subglottic FB. It was a hollow plastic tube facing end on. Spontaneous ventilation was maintained throughout the procedure and $100 \% \mathrm{O}_{2}$ was insufflated using an open circuit. The child did not cough or buck. The surgeon grasped the FB circumferentially using an optical forceps but was unsuccessful in retrieving the $\mathrm{FB}$ as the entire assembly was too large to negotiate the glottis. Inadequate relaxation of the cords was suggested as a possible cause for the difficulty. A rigid bronchoscope was inserted and the FB was maneuvered into the right bronchus. After ensuring the effectiveness of ventilation through the ventilating port of the bronchoscope, neuromuscular blockade was achieved with atracurium $10 \mathrm{mg}$ intravenously and the propfol infusion was stepped down to $6 \mathrm{mg} / \mathrm{kg} /$ hour. Two minutes later, another attempt at FB retrieval was made but this was also futile. The vitals remained stable with a heart rate of $120 /$ minute, BP $96 / 60 \mathrm{mmHg}$ and saturation $98-100 \%$. Keeping all equipment ready for tracheotomy, a third attempt was made and this time the rim of the cylindrical FB was held between the limbs of the optical forceps (alligator) and it was successfully removed in total. Examination of the FB (Figure 1) showed that it consisted of two plastic tubes placed coaxially within each other and both had been removed together. A check bronchoscopy was done to identify any retained piece. The rigid bronchoscope was removed and the trachea intubated with $4.5 \mathrm{~mm}$ internal diameter cuffed endotracheal tube to enable mechanical ventilation and tracheobronchial suctioning. The child showed signs of spontaneous respiratory efforts around 20 minutes later. Neuromuscular blockade was reversed and trachea was extubated after giving intravenous lidocaine $20 \mathrm{mg}$ to prevent laryngospasm. Post extubation, he had severe cough and mild bronchospasm, both of which were managed with budesonide nebulisation. The child was awake and alert with a respiratory rate of $24 /$ minute, heart rate 120/minute and SpO2 $98-100 \%$ on oxygen therapy. The child also received epinephrine nebulisation. His dysphonia improved and he was discharged the next day.

\section{Discussion}

The whistle was found to be the most common non organic and second most common tracheobronchial FB [1]. Symptoms of a subglottic FB include cough which might subside after sometime due to fatigue and adaptation [2]. Stridor and dysphonia are classical symptoms of a laryngotracheal FB. A witnessed choking episode is the most reliable sign of a tracheobronchial FB requiring urgent investigation and management [3]. Clinical examination and roentgenogram of the neck and chest are the investigations required. 


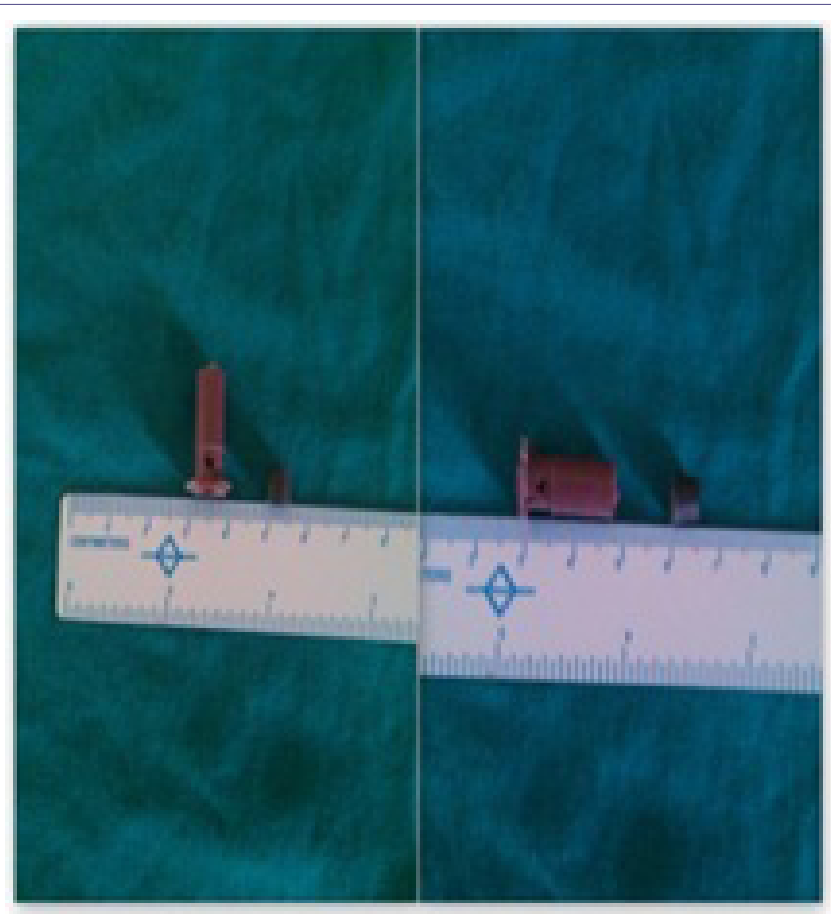

Figure 1: Whistle - the subglottic foreign body.

Plastic laryngeal FB often have a delayed diagnosis in view of their radiolucency and late onset obstructive emphysema [3]. Delayed detection and management $>7$ days results in greater morbidity including mucosal edema, bleeding and granuloma [4]. In this patient the only symptom was dysphonia since the choking episode. It did not cause any respiratory distress as its hollow cylindrical disposition mimicked an endotracheal tube and allowed inspiration and expiration through its lumen. Other types of foreign bodies may exert a ball valve effect causing bilateral hyperinflation of the chest [5].

Some patients with a large FB causing respiratory distress may require urgent tracheostomy to relieve airway obstruction [6]. Tracheostomy may also be required to remove subglottic FB larger than the laryngeal inlet to avoid vocal cord injury, sharp objects whose retrieval may lacerate the cords and long standing FB with granulation tissue that may bleed [7].

FB which is clearly visible on direct laryngoscopy, smaller than the glottic chink and not adherent may be removed atraumatically using a Magill's forceps or optical forceps [8]. Khan et al., described 220 cases of whistle as a foreign body of which $15 \%$ were laryngotracheal, and all were successfully removed by rigid bronchoscopy [9].

It is essential to maintain spontaneous ventilation in all cases of suspected subglottic FB as loss of spontaneous ventilation may prove fatal in the event of total airway obstruction. Neuromuscular blockade may be established once it has been confirmed that ventilation through the ventilating port of the bronchoscope is possible either with the FB in situ or after it has been pushed distally into the right bronchus. Controlled ventilation reduces the anaesthetic requirement, improves ventilation, and decreases coughing or bucking during the procedure [10].

It is helpful to know the construction of non animate foreign bodies like whistle which may consist of three parts as in this case. The third bit was a transparent portion which had been coughed out the earlier day. It is necessary to carefully fit the pieces externally to see if they have been removed completely. Transparent plastic or glass pieces are more likely to be missed if not specifically looked for [6].

In conclusion, it may be said that subglottic foreign bodies require urgent bronchoscopy. Preparation for emergent tracheotomy needs to be ready before starting the case and spontaneous ventilation should not be abolished until effective ventilation through the ventilating bronchoscope is confirmed.

\section{References}

1. Mukherjee M, Paul R (2011) Foreign body aspiration: demographic trends and foreign bodies posing a risk. Indian J Otolaryngol Head Neck Surg 63: 313-316.

2. Kaur K, Sonkhya N, Bapna AS (2002) Foreign bodies in the tracheobronchial tree: A prospective study of fifty cases. Indian J Otolaryngol Head Neck Surg 54: 30-34.

3. Bloom DC, Christenson TE, Manning SC, Eksteen EC, Perkins JA, et al. (2005) Plastic laryngeal foreign bodies in children: a diagnostic challenge. Int J Pediatr Otorhinolaryngol 69: 657-662.

4. Chik KK, Miu TY, Chan CW (2009) Foreign body aspiration in Hong Kong Chinese children. Hong Kong Med J 15: 6-11.

5. Kenth J, Ng C (2013) Foreign body airway obstruction causing a ball valve effect. JRSM Short Rep 4: 2042533313482458.

6. Anju G, Raman W, Sarla H, Kirti K, Susheela T, et al. (2009) Unusual Subglottic Foreign Bodies. J Anaesth Clin Pharmacol 25: 376-377.

7. Singh JK, Vasudevan V, Bharadwaj N, Narasimhan KL (2009) Role of tracheostomy in the management of foreign body airway obstruction in children. Singapore Med J 50: 871-874.

8. Gupta K, Gupta PK (2010) Laryngoscopic removal of unusual metallic foreign body of the subglottic region of the larynx. Anesth Essays Res 4: 106-108.

9. Khan AR, Khan NS (2004) Management of whistle as a foreign body tracheobronchial tree. Journal of Postgraduate Medical Institute 18: 602-606.

10. Litman RS, Ponnuri J, Trogan I (2000) Anesthesia for tracheal or bronchial foreign body removal in children: an analysis of ninety-four cases. Anesth Analg 91: 1389-1391. 